\title{
O uso de modelagem de distribuição de espécies para restauração florestal: Uma
}

\section{revisão sistemática}

\author{
The use of species distribution modeling for forest restoration: A systematic review \\ El uso del modelado de distribución de especies para la restauración forestal: Una revisión \\ sistemática
}

Recebido: 14/06/2021 | Revisado: 24/06/2021 | Aceito: 05/07/2021 | Publicado: 16/07/2021

\author{
Luise Andrade Amaral \\ ORCID: https://orcid.org/0000-0003-4497-6542 \\ Universidade Federal de Sergipe, Brasil \\ E-mail: andrade.luise@ hotmail.com \\ Robério Anastácio Ferreira \\ ORCID: https://orcid.org/0000-0003-4436-583X \\ Universidade Federal de Sergipe, Brasil \\ E-mail: roberioaf@yahoo.com.br \\ Renata Silva Mann \\ ORCID: https://orcid.org/0000-0001-5993-3161 \\ Universidade Federal de Sergipe, Brasil \\ E-mail: renatamann@academico.ufs.br
}

\begin{abstract}
Resumo
O objetivo deste trabalho foi realizar uma revisão sistemática da produção científica do uso da modelagem de distribuição de espécies para restauração florestal. As buscas de artigos científicos nas bases de dados Scopus e Web of Science para os últimos 15 anos foram realizadas no mês de dezembro de 2020 utilizando os termos: "ecological modeling" OR "biodiversity modeling" OR "predictive models" OR "niche modeling" OR "habitat models" AND "species distribution" OR "geographic distribution" OR "potential distribution" AND "forest restoration" OR "restoration ecology". Para as análises estatísticas e gráficos dos dados brutos foi utilizado o pacote Bibliometrix do software R. Os dados brutos foram refinados por meio da seleção dos estudos que atenderam aos seguintes critérios: (i) estudos publicados em revistas científicas com fator de impacto igual ou superior a 2,0; (ii) estudos em que o título ou resumo mencionasse as palavras restauração florestal ou restauração ecológica; (iii) estudos que avaliaram o uso de modelagem de distribuição de espécies como auxílio aos projetos e programas de restauração florestal ou restauração ecológica. Foram encontrados 44 documentos publicados em 30 periódicos científicos com média de 3,91 publicações por ano; 18,55 citações por documento; 197 autores, sendo 3 documentos com autoria única. Assim pode-se concluir que o uso de modelagem de distribuição de espécies para restauração florestal no mundo é muito recente, e no Brasil é incipiente com baixos números de artigos publicados, mas apresenta tendência de crescimento por conta da sua significativa contribuição para melhorar as taxas de sucesso dos projetos de restauração.
\end{abstract}

Palavras-chave: Restauração Ecológica, Maxent, Seleção de Espécies.

\begin{abstract}
The objective of this work was to carry out a systematic review of the scientific production on the use of species distribution modeling for forest restoration. Searches for scientific articles in the Scopus and Web of Science databases for the last 15 years were performed in December 2020 using the terms: "ecological modeling" OR "biodiversity modeling" OR "predictive models" OR "modeling of niche" OR "habitat models" AND "species distribution" OR "geographic distribution" OR "potential distribution" AND "forest restoration" OR "restoration ecology". For the statistical and graphical analysis of the raw data the Bibliometrix package was used of the R software. The raw data were refined by selecting the studies that meet the following criteria: (i) studies published in scientific journals with an impact factor equal to or greater than 2.0; (ii) studies in which the title or abstract mentioned as words forest restoration or ecological restoration; (iii) studies that evaluate the use of species distribution modeling as an aid to forest restoration or restoration projects and programs the ecological. found 44 documents published in 30 scientific journals with an average of 3.91 publications per year; 18.55 citations per document; 197 authors, 3 documents with single authorship. Thus, we were able to conclude that the use of species distribution modeling for forest restoration in the world is very recent, and in Brazil it is incipient with low numbers of published articles. Still, it shows a growing trend due to its significant contribution to improving success rates restoration projects.
\end{abstract}

Keywords: Ecological Restoration, Maxent, Species Selection. 


\begin{abstract}
Resumen
El objetivo de este trabajo fue realizar una revisión sistemática de la producción científica sobre el uso de modelos de distribución de especies para la restauración forestal. Las búsquedas de artículos científicos en las bases de datos de Scopus y Web of Science durante los últimos 15 años se llevaron a cabo en diciembre de 2020 utilizando los términos: "modelado ecológico" O "modelado de biodiversidad" O "modelos predictivos" O "modelado de hábitat de nicho" O " modelos "Y" distribución de especies "O" distribución geográfica "O" distribución potencial "Y" restauración forestal "O" ecología de restauración ". Para el análisis estadístico y gráfico de los datos brutos se utilizó el paquete Bibliometrix del software R. Los datos se refinaron seleccionando los estudios que cumplen con los siguientes criterios: (i) estudios publicados en revistas científicas con un factor de impacto igual o superior a 2.0; (ii) estudios en los que el título o resumen menciona como palabras restauración forestal o restauración ecológica; (iii) estudios que evalúen el uso de modelos de distribución de especies como ayuda para proyectos y programas de restauración o restauración forestal lo ecológico. encontró 44 documentos publicados en 30 revistas científicas con un promedio de 3.91 publicaciones por año; 18,55 citas por documento; 197 autores, 3 documentos de autoría única. Así, podríamos concluir que el uso de modelos de distribución de especies para la restauración forestal en el mundo es muy reciente, y en Brasil es incipiente con bajo número de artículos publicados, pero muestra una tendencia creciente debido a su significativa contribución a mejorar las tasas de éxito. proyectos de restauración.
\end{abstract}

Palabras clave: Restauración Ecológica, Maxent, Selección de Especies.

\title{
1. Introdução
}

À medida que as condições climáticas mudam, também são alteradas as distribuições potenciais das espécies, ou seja, mudam-se aquelas áreas da paisagem com condições adequadas para a persistência de uma espécie. No entanto, a compreensão de como as espécies serão impactadas é limitada, dada a complexidade das mudanças climáticas e a variedade de escalas em que seus efeitos ocorrerão (Renton et al. 2012). Assim, há necessidade de intensificar os esforços de pesquisa em áreas ameaçadas por mudanças globais e criar ações para reduzir seus efeitos (Hulme 2009; Richardson; Rejmánek 2011; Lehmann et al. 2017; Meira-Neto et al. 2017).

A modelagem preditiva de distribuição de espécies é um método computacional que permite uma representação das condições requeridas para a sobrevivência de uma ou mais espécies, por meio da combinação de dados de ocorrência com variáveis ambientais (Anderson et al. 2003). Esses modelos têm a capacidade de prever a distribuição futura da vegetação sob diferentes cenários de mudanças climáticas (Leach et al. 2016; Virgili et al.2017), avaliar a eficácia das Áreas Protegidas (Ferro et al. 2014; Lemes et al. 2014), procurar ocorrências de novas populações de espécies ameaçadas (Kamino et al. 2012; Pena et al. 2014; Cupertino-Eisenlohr et al. 2017; Oliveira et al. 2016, 2017) e têm sido considerados ferramentas essenciais para a gestão e conservação da biodiversidade (Cote \& Reynolds, 2002).

Além disso, os modelos de distribuição de espécies (SDM) podem prever áreas para restauração, translocação ou reintroduções assim como prever as espécies mais adequadas para um determinado local (Araújo \& Peterson, 2012). Com base na área de adequação, gestores de terras e formuladores de políticas podem estabelecer mecanismos para identificar áreas de alto risco de invasão, onde a localização de áreas para restauração deve ser priorizada (Allen \& Bradley, 2016). Apesar das atividades de restauração ecológica demandarem custos elevados e não oferecem retorno financeiro direto para os proprietários rurais, seus benefícios são de longo prazo, coletivos e difusos (Costa, 2016). Contudo, muitos fatores como, a dispersão de sementes invasivas, bancos de sementes e mudas, conectividade da paisagem, limitações socioeconômicas e invasão biológica podem comprometer o seu sucesso (Melo et al. 2013; Martinez-Ramos et al. 2016).

Desta forma, os esforços para restaurações futuras devem ser direcionados para o estabelecimento de ecossistemas que são capazes de persistir sob novas condições (Cairns, 2002; Choi, 2004; Gastón \& García-Viñas, 2013), uma vez que a gestão das mudanças climáticas requer estratégias de curto e longo prazo que permitirão maior resistência e resiliência nos ecossistemas (Millar et al. 2007). Neste sentido, a resposta das espécies às mudanças ambientais pode afetar os resultados da restauração principalmente por ser um processo de impacto de longo prazo (Harris et al. 2006). Se as espécies selecionadas não podem tolerar as modificações ambientais previstas pelas mudanças climáticas, a estratégia de restauração não funcionará a médio e longo prazo. Até o momento, apenas as condições climáticas históricas e atuais são levadas em consideração no 
estabelecimento dos requisitos necessários para projetos de restauração (Ravenscroft et al. 2010).

As primeiras aplicações de SDM para apoiar a seleção de espécies para iniciativas de restauração florestal utilizaram envelopes ambientais para estimar a adequação específica do local (Gutiérrez \& Sánchez Palomares; 1994). Em seguida métodos de modelagem mais complicados como a abordagem de casco convexo (Garcia López \& Allúe Camacho, 2004) e os modelos lineares generalizados (Felicisimo, 2003) passaram a ser utilizados para ajustar SDM que apoiam a seleção de espécies na restauração florestal (Gaston et al. 2014). Porém, mesmo com elevado potencial de aplicabilidade para restauração florestal (Gastón et al. 2014), os SDM raramente foram aplicados para esta finalidade (Burnside et al. 2002; Van Loon et al. 2011; Gastón et al. 2014) talvez pela necessidade de integração de níveis hierárquicos (Gastón \& García-Viñas, 2010), ou pela escolha de uma amostragem apropriada (Mateo et al. 2018).

Sendo assim, uma alternativa para alcançar melhores resultados poderia ser a adoção de uma estratégia ganha-ganha (Brancalion \& Chazdon, 2017), priorizando ações que mitiguem mais de um fator negativo, como a promoção da restauração em áreas com alto risco de invasão biológica que aumentará a probabilidade de sucesso da restauração e reduzirá a disponibilidade de habitat para espécies invasoras (Heringer, Bueno, Meira-Neto, Matos \& Neri, 2019). Portanto, este estudo teve como objetivo realizar uma revisão sistemática sobre o uso da modelagem de distribuição de espécies para restauração florestal.

\section{Metodologia}

Uma revisão sistemática é um resumo conciso das melhores evidências disponíveis que usa métodos explícitos e rigorosos para identificar, avaliar criticamente e sintetizar estudos relevantes sobre um tópico específico (Dybå \& Dingsøyr, 2008). Além de ser baseado em uma estratégia de busca abrangente e detalhada, capaz de identificar limites em torno de generalizações e importantes lacunas geográficas permite estudos teóricos e metodológicos na literatura (Pickering \& Bryne, 2014; Torraco, 2016).

Com este estudo realizou-se buscas de artigos científicos nas bases de dados Scopus e Web of Science para os últimos 15 anos, ou seja, de 2005 a 2020. Essas buscas foram realizadas no mês de dezembro de 2020 utilizando os termos: "ecological modeling" OR "biodiversity modeling" OR "predictive models" OR "niche modeling" OR "habitat models" AND "species distribution" OR "geographic distribution" OR "potential distribution" AND "forest restoration" OR "restoration ecology".

Para a realização das análises estatísticas e gráficos foi utilizado o pacote Bibliometrix do software R (R CORE TEAM, 2020) que é uma ferramenta de código aberto para executar uma abrangente análise de mapeamento científico da literatura cientifica (Ariaa \& Cuccurullo, 2017). Para isso os dados bibliográficos (brutos) foram importados no formato BibTex para realização de análises bibliométricas como, citações, colaboração científica e análise de artigos por ano de publicação.

Os dados brutos foram refinados por meio da seleção dos estudos que atenderam aos seguintes critérios: (i) estudos publicados em revistas científicas com fator de impacto igual ou superior a 2,0; (ii) estudos em que o título ou resumo mencionasse as palavras restauração florestal ou restauração ecológica; (iii) estudos que avaliaram o uso de modelagem de distribuição de espécies como auxílio aos projetos e programas de restauração florestal ou restauração ecológica.

Desta forma, este processo de filtragens sucessivas constituiu um fluxograma de pesquisa (Figura 1), conforme requerido pela instrução QUOROM, a fim de facilitar a visualização do porquê os estudos foram excluídos em diferentes estágios da revisão e por quais diferentes razões isto aconteceu (Dybå \& Dingsøyr, 2008). 
Figura 1: Protocolo de seleção dos artigos.

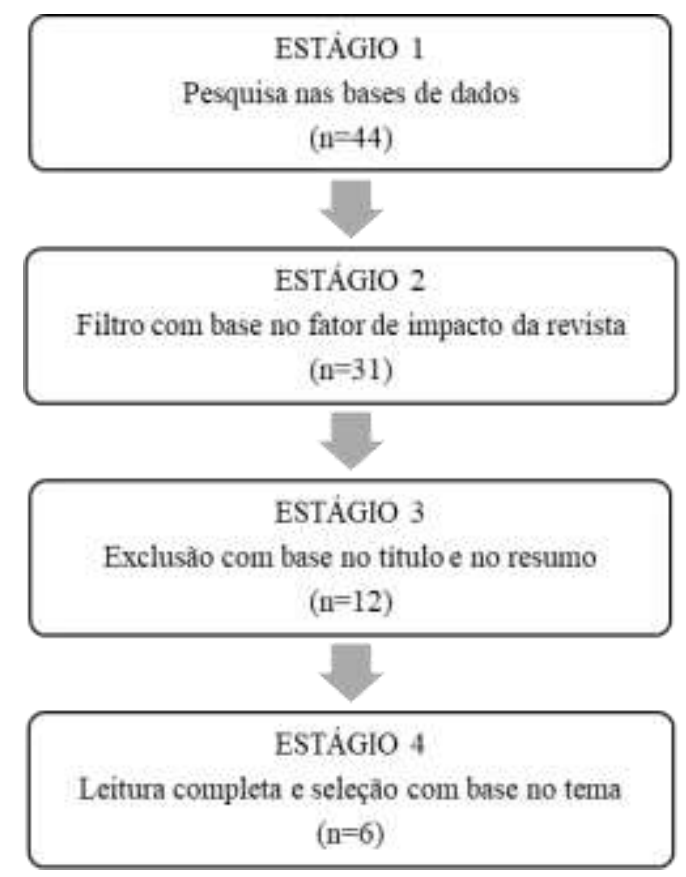

Fonte: Adaptado de Dybå \& Dingsøyr (2008).

\section{Resultados e Discussão}

Esta pesquisa totalizou 45 artigos, sendo 1 identificado como duplicata, restando 44 artigos publicados em 30 periódicos científicos com média de 3,91 publicações por ano. Dentre as fontes mais relevantes as revistas "Biodiversity and Conservation", "Biological Invasions", "Ecological Engineering”, "Forest Ecology and Management" e "Journal of Applied Ecology" apresentaram o mesmo número de publicações $(n=3)$. Foram contabilizadas 18,55 citações por documento e 197 autores, sendo 3 documentos com autoria única.

Apesar do intervalo de buscas ter sido definido para 15 anos, ou seja, de 2005 a 2020 não foram encontradas publicações antes do ano 2010, assim, o intervalo de publicações passou a ser de 10 anos com média de 3,92 artigos por ano, com 10 artigos $(22,7 \%)$ publicados somente no ano de 2018 (Figura 2).

Figura 2: Número de publicações científicas anuais.

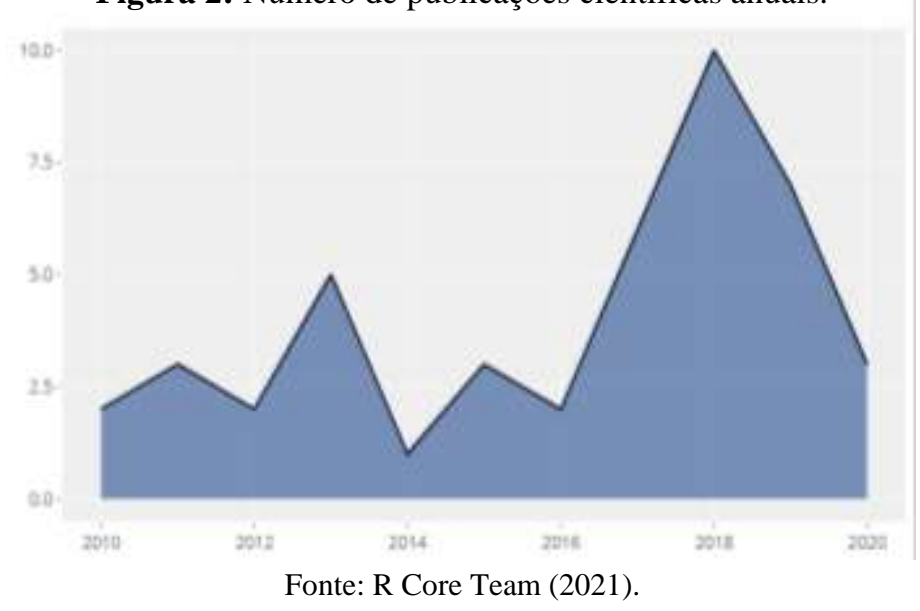


Os Estados Unidos é o país com maior número de trabalhos publicados sobre o uso de SDM para restauração florestal $(\mathrm{n}=11)$, seguido da Espanha $(\mathrm{n}=5)$, da Austrália e do Brasil $(\mathrm{n}=4)$ e da China $(\mathrm{n}=3)$. Desta forma, o Brasil ocupa a $3^{\mathrm{a}}$ posição juntamente com a Austrália ambos com 9,1\% dos documentos publicados (Figura 3). No entanto, dos três artigos brasileiros apenas um teve como objetivo investigar como o efeito de condições climáticas atuais e futuras na distribuição geográfica Acacia mangium e Acacia auriculiformis na Mata Atlântica impactaria os programas de restauração florestal desse bioma, visto que são espécies exóticas que podem invadir áreas potenciais para restauração (Heringer et al. 2019).

Figura 3: Distribuição geográfica das publicações científicas encontradas.

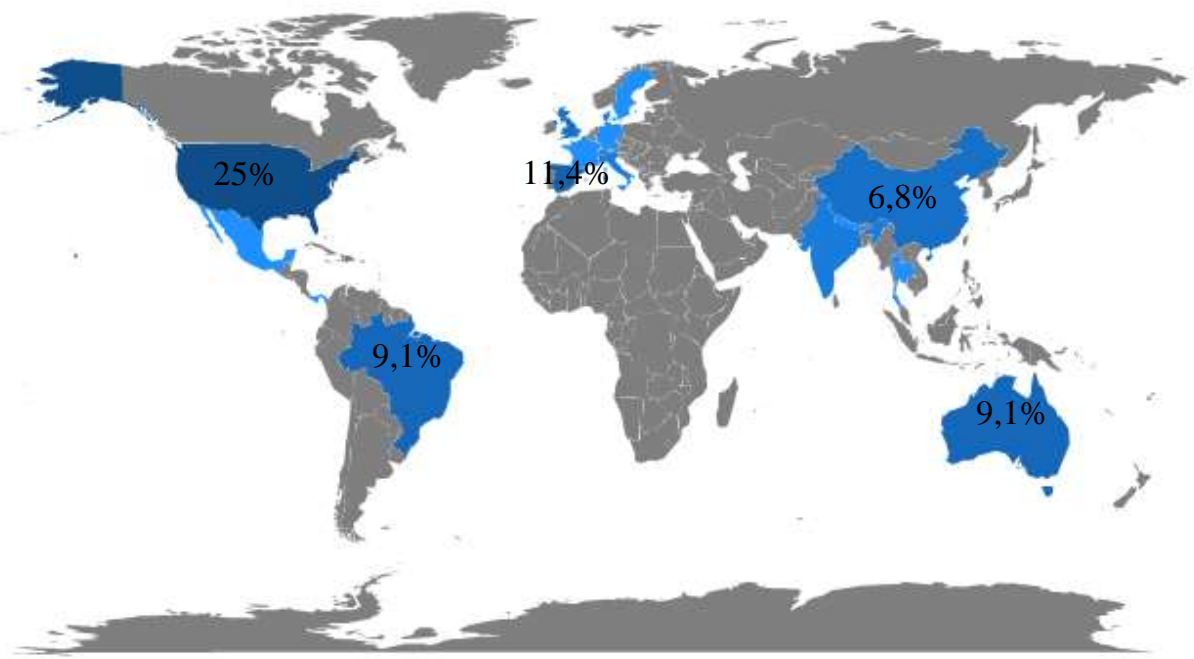

Fonte: R Core Team (2021).

$\mathrm{O}$ artigo que apresentou o maior número de citações $(\mathrm{n}=145)$ também corresponde ao primeiro artigo publicado dentro do espaço de tempo selecionado nas buscas. Este artigo mais citado tem como objetivo o uso de modelagem para avaliar o habitat climático atual ou terras climaticamente adequadas para invasão de três espécies invasoras no sudeste dos Estados Unidos (Bradley et al., 2010) que foi o país com maior número de citações ( $=203$ ) seguido da Austrália $(\mathrm{n}=200)($ Figura 4).

Figura 4: Comparação entre os documentos e países mais citados.
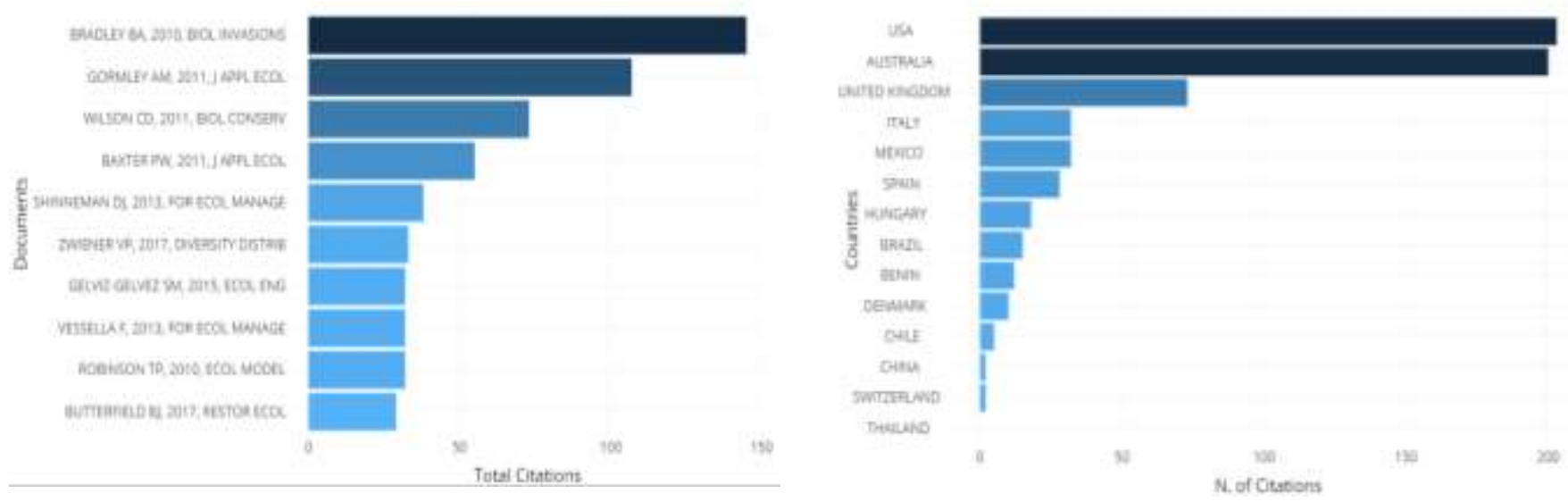

Fonte: R Core Team (2021).

Após as análises estatísticas realizadas para todos os documentos encontrados $(\mathrm{n}=44)$ foram realizadas filtragens específicas para selecionar os artigos que tratavam diretamente do uso de SDM na restauração florestal. O primeiro filtro aplicado foi em relação ao fator de impacto das revistas, ou seja, foram excluídos os artigos publicados em revistas com fator 
de impacto menor que 2, uma vez que quanto maior o fator de impacto mais prestígio acadêmico a revista possui. Assim, restaram 31 artigos dos quais foram selecionados aqueles que continham nos títulos ou resumos as palavras "restauração florestal" ou "restauração ecológica" o que totalizou 12 artigos. Foi feita a leitura a fim de selecionar àqueles que avaliavam o uso da modelagem de distribuição de espécies na restauração florestal, sendo selecionados 6 artigos (Tabela 1).

Tabela 1: Lista dos artigos selecionados para análise conforme critérios empregados no protocolo de pesquisa. Onde: NC = número de citações

\begin{tabular}{|c|c|c|c|c|}
\hline AUTORES & REVISTA & ANO & PAÍS & NC \\
\hline Gaston A; Garcia-Vinas J & Ecological Modelling & 2013 & Espanha & 15 \\
\hline $\begin{array}{l}\text { Gaston A; Garcia-Vinas J; Bravo-Fernandez A; } \\
\text { Lopez-Leiva C; Oliet J; Roig S; Serrada R }\end{array}$ & New Forests & 2014 & Espanha & 13 \\
\hline $\begin{array}{c}\text { Gelviz-Gelvez S; Pavn N; Illoldi-Rangel P; } \\
\text { Ballesteros-Barrera C }\end{array}$ & Ecological Engineering & 2014 & México & 32 \\
\hline Cai A; Wang J; Wang Y; Maclachlan I & Environmental Earth Sciences & 2019 & China & 1 \\
\hline Heringer G; Bueno M; Meira-Neto J; Matos F; Neri A & Biological Invasions & 2019 & Brasil & 3 \\
\hline Carrasco J; Price V; Tulloch V; Mills M & Biodiversity and Conservation & 2020 & África & 0 \\
\hline
\end{tabular}

Fonte: Autores (2021).

O intervalo de publicações para os 6 artigos selecionados (Tabela 1) foi de 7 anos com o primeiro artigo publicado em 2013 (Gastón \& García-Viñas, 2013) e o último em 2020 (Carrasco, Price, Tulloch \& Mills, 2020) tendo como média de publicação 1,2 artigo por ano. Destes o artigo mais citado foi aquele desenvolvido no México (Gelviz-Gelvez, Pavn, IlloldiRangel \& Ballesteros-Barrera, 2014), seguido dos que foram desenvolvidos na Espanha (Gastón \& García-Viñas, 2013; Gaston et al. 2014) conforme descrito na Tabela 1.

De acordo com a Society for Ecological Restoration International [SER] (2004), a restauração ecológica é uma atividade intencional que inicia ou acelera a recuperação de um ecossistema degradado em relação a sua saúde, integridade e sustentabilidade. Em todo o mundo, as estratégias mais frequentes para restauração são: plantio de mudas e semeadura direta (Florentine \& Westbrooke, 2004) o que faz a seleção adequada de espécies obrigatória para os projetos de restauração (Gastón \& García-Viñas, 2013). No entanto, muitos critérios diferentes podem ser usados na seleção de espécies, devendo as espécies selecionadas serem combinadas com as condições ambientais locais (Peman Garcia et al. 2008). Deve-se considerar que a estimativa de adequação do habitat para as espécies selecionadas parte dos resultados dos modelos preditivos de distribuição de espécies (SDMs), que relacionam observações de campo com variáveis preditoras ambientais (Guisan \& Thuiller, 2005).

Os protocolos de modelagem envolvem a utilização de ferramentas de Sistemas de Informações Geográficas (SIGs), softwares e algoritmos de modelagem (Cancian, 2012). Neste sentido, para ajustarem os modelos de distribuição de espécies, 50\% $(\mathrm{n}=3)$ dos artigos selecionados utilizaram o software MaxEnt (Gelviz-Gelvez et al. 2014; Heringer et al. 2019; Carrasco et al. 2020) por ser baseado no princípio da máxima entropia (Phillips et al. 2006), a regressão logística penalizada (Harrell, 2001) foi a escolha de 33,3\% dos artigos (Gastón \& García-Viñas, 2013; Gaston et al. 2014) e os modelos aditivos generalizados (GAMs) foi a opção de somente um (16,67\%) dos artigos (Cai, Wang, Wang \& Maclachlan, 2019).

$\mathrm{O}$ algoritmo MaxEnt tem sido amplamente utilizado em modelagem de distribuição de espécies por apresentar resultados robustos quando comparado a outros algoritmos de modelagem, mesmo quando utilizado com um número reduzido de dados de ocorrência da(s) espécie(s) estudada(s). Com este algoritmo busca-se encontrar a distribuição mais próxima da distribuição uniforme baseada nas restrições que as variáveis disponíveis relacionadas à observação de uma espécie e as condições ambientais de determinada área geográfica oferecem ao modelo (Cancian, 2012). 
Dos artigos que utilizaram o MaxEnt podemos destacar Gelviz-Gelvez et al. (2014) que foi realizado com o objetivo de selecionar espécies arbustivas com atributos ecológicos (cobertura, densidade, frequência, associação coeficiente e presença de associações micorrízicas) que permitam a rápida recuperação de ecossistemas semiáridos perturbados. Tendo como resultados a coexistência de ambos os grupos funcionais de arbustos (perene e decíduo), mas com a recomendação de seis espécies (Acacia schaffneri, Ageratina espinosarum, Bursera fagaroides, Dalea bicolor, Eysenhardtia polystachya $e$ Karwinskia humboldtiana) que apresentaram qualidades ecológicas adequadas para restauração e manutenção ou ampliação de sua distribuição no cenário de mudanças climáticas de médio e longo prazo na região semiárida do México Central.

Outro destaque é o artigo realizado no Brasil, que também utilizou o MaxEnt, só que com o objetivo de investigar o efeito de condições climáticas presente e futuro na distribuição geográfica de espécies invasoras (Acacia mangium e Acacia auriculiformis) na Mata Atlântica, para buscar entender o possível impacto desta distribuição nos programas de restauração florestal desse bioma, visto que são espécies exóticas que podem invadir áreas potenciais para restauração. Os autores concluíram que há na Mata Atlântica uma grande área mais adequada para a espécie A. mangium, do que para A. auriculiformis sugerindo que este bioma pode sofrer um fator adicional de degradação nos anos seguintes e que os esforços para restauração local podem ser impedidos por invasão biológica (Heringer et al. 2019).

Desta forma, é possível afirmar que a etapa de escolha de espécies para projetos de restauração florestal encontrou na modelagem de distribuição preditiva uma grande aliada, pois seus modelos podem e devem diminuir o risco de seleção de espécies inadequadas e, consequentemente, melhorar as taxas de sucesso dos projetos de restauração (Gastón \& García-Viñas, 2013). No Brasil os trabalhos com modelagem de distribuição de espécies arbóreas estavam restritos ao bioma Mata Atlântica (Giacomin et al. 2014) Porém, em estudo para determinar a distribuição potencial de 23 espécies pioneiras no Estado de Minas Gerais, os autores concluíram que a modelagem preditiva de distribuição indica espécies mais adequadas para cada bioma existente no Estado (Caatinga, Cerrado e Mata Atlântica), e permite relacionar as características do bioma às preferências ambientais de cada espécie (Coelho, Carvalho \& Gomide, 2016).

Acredita-se, portanto, que a recomendação para utilização do Maxent como ferramenta para orientar os planos de conservação que necessitam de indicação de espécies, para recuperar áreas de vegetação degradadas ou desmatadas (Coelho, Carvalho \& Gomide, 2016) se dá porque este algoritmo calcula a maioria da distribuição geográfica potencial de uma espécie, principalmente a partir da relação entre os dados e a distribuição geográfica conhecida para as espécies trabalhando apenas com dados de presença e presença-ausência (Elith et al. 2011).

\section{Conclusão}

Diante dos resultados obtidos, pode-se concluir que o uso de modelagem de distribuição de espécies para restauração florestal no mundo é muito recente, mas apresenta tendência de crescimento por conta da sua significante contribuição para melhorar as taxas de sucesso dos projetos de restauração, uma vez que auxilia na escolha das espécies florestais mais adaptadas tanto ao local quanto as mudanças climáticas podendo servir como aliado na prevenção de invasão biológica.

No caso do Brasil, pode-se concluir que o tema ainda é incipiente com baixos números de artigos publicados, mas com boas contribuições que servem de base para tomadas de decisão em futuros projetos de restauração florestal localizados, principalmente, em áreas do bioma Mata Atlântica. Assim, percebe-se há necessidade de inclusão da modelagem de distribuição de espécies para os demais biomas brasileiros.

É importante salientar que devido aos termos de busca e filtros aplicados neste estudo os resultados aqui obtidos são restritivos e refletem uma parte da totalidade de estudos realizados sobre o uso da modelagem de distribuição de espécies nos programas de restauração florestal tanto internacional como nacional. 


\section{Referências}

Allen, J. M., \& Bradley, B. A. (2016). Out of the weeds? Reduced plant invasion risk with climate change in the continental United States. Biological Conservation, 203, 306-312. https://doi.org/10.1016/j.biocon.2016.09.015

Anderson, R. P., Lew, D., \& Peterson, A. T. (2003). Evaluating predictive models of species' distributions: criteria for selecting optimal models. Ecological Modelling, 162(3), 211-232. https://doi.org/10.1016/S0304-3800(02)00349-6

Araújo, M. B. \& Peterson, A. T. 2012. Uses and misuses of bioclimatic envelope modelling. Ecology, 93: 1527-1539. https://doi.org/10.1890/11-1930.1

Aria, M., \& Cuccurullo, C. (2017). bibliometrix: An R-tool for comprehensive science mapping analysis. Journal of informetrics, 11(4), 959-975. https://doi.org/10.1016/j.joi.2017.08.007

Bradley, B. A., Wilcove, D. S., \& Oppenheimer, M. (2010). Climate change increases risk of plant invasion in the Eastern United States. Biological Invasions, 12(6), 1855-1872. https://doi.org/10.1007/s10530-009-9597-y

Brancalion, P. H., \& Chazdon, R. L. (2017). Beyond hectares: four principles to guide reforestation in the context of tropical forest and landscape restoration. Restoration Ecology, 25(4), 491-496. https://doi.org/10.1111/rec.12519

Brearley, F. Q. (2011) Below-Ground secondary succession in tropical forests of borneo. Journal of Tropical Ecology, 27, 413-420. https://doi.org/10.1017/S0266467411000149

Burnside, N. G., Smith, R. F., \& Waite, S. (2002). Habitat suitability modelling for calcareous grassland restoration on the South Downs, United Kingdom. Journal of Environmental Management, 65(2), 209-221. https://doi.org/10.1006/jema.2002.0546

Cai, A., Wang, J., Wang, Y., Maclachlan, I. (2019) Spatial optimizations of multiple plant species for ecological restoration of the mountainous areas of north china. Environmental Earth Sciences, 78: 302. https://doi.org/10.1007/s12665-019-8299-8

Cairns, J., 2002. Rationale for restoration. In: Handbook of Ecological Restoration: Vol. 1. Principles of Restoration. Cambridge University Press, Cambridge, UK.

Cancian, L. F. (2012) Modelagem de distribuição geográfica potencial de macrófitas aquáticas em bacias hidrográficas. Tese de doutorado, Universidade Estadual Paulista, Instituto de Biociências de Rio Claro, 2012. Disponível em: 〈http://hdl.handle.net/11449/100619>.

Carrasco, J., Price, V., Tulloch, V., Mills, M. (2020) Selecting priority areas for the conservation of endemic trees species and their ecosystems in Madagascar considering both conservation value and vulnerability to human pressure. Biodiversity and Conservation, 29, 1841-1854. https://doi.org/10.1007/s10531-02001947-1

Choi, Y. D., (2004) Theories for ecological restoration in changing environment: toward "futuristic" restoration. Ecological Research, 19, 75-81. https://doi.org/10.1111/rec.13265

Coelho, G. L. N., Carvalho, L. M. T. D., \& Gomide, L. R. (2016). Modelagem preditiva de distribuição de espécies pioneiras no Estado de Minas Gerais. Pesquisa Agropecuária Brasileira, 51(3), 207-214. DOI:10.1590/S0100-204X2016000300002

Costa, M. M. (2016) Financiamento para a restauração ecológica no Brasil. Mudanças no Código Florestal Brasileiro: desafios para implementação da nova lei, [S. 1.], p. 235-260.

Cote, I., Reynolds, J., (2002) Predictive ecology to the rescue? Science, vol. 298, pp. 1181-1182. DOI:10.1126/science.1079074

Cupertino-Eisenlohr, M. A., Vinícius-Silva, R., Meireles, L. D., Eisenlohr, P. V., Meira-Neto, J. A., \& Santos-Gonçalves, A. P. (2017). Stability or breakdown under climate change? A key group of woody bamboos will find suitable areas in its richness center. Biodiversity and Conservation, $26(8), 1845-1861$. https://doi.org/10.1007/s10531-017-1332-x

de Castro Pena, J. C., Kamino, L. H. Y., Rodrigues, M., Mariano-Neto, E., \& de Siqueira, M. F. (2014). Assessing the conservation status of species with limited available data and disjunct distribution. Biological Conservation, 170, 130-136. https://doi.org/10.1016/j.biocon.2013.12.015

Dybå, T., \& Dingsøyr, T. (2008, October). Strength of evidence in systematic reviews in software engineering. In Proceedings of the Second ACM-IEEE international symposium on Empirical software engineering and measurement (pp. 178-187). https://doi.org/10.1145/1414004.1414034

Felici`Simo, A.M. (2003) Uses of spatial predictive models in forested areas territorial planning. Ciot-IV International Conference on Spatial Planning, pp 115.

Ferro, V. G., Lemes, P., Melo, A. S., \& Loyola, R. (2014). The reduced effectiveness of protected areas under climate change threatens Atlantic Forest tiger moths. PLoS One, 9(9), e107792. https://doi.org/10.1371/journal.pone.0107792

Florentine, S. K., \& Westbrooke, M. E. (2004). Restoration on abandoned tropical pasturelands-do we know enough?. Journal for Nature Conservation, 12(2), 85-94. https://doi.org/10.1016/j.jnc.2003.08.003

García-López, J. M., \& Camacho, C. A. (2005, June). Ensayo de un sistema fitoclimático de carácter autoecológico para especies arbóreas forestales en la península ibérica y su aplicación en labores de repoblación forestal. In Congresos Forestales.

G Gaston, A., \& Garcia-Vinas, J. I. (2013). Evaluating the predictive performance of stacked species distribution models applied to plant species selection in ecological restoration. Ecological Modelling, 263, 103-108. https://doi.org/10.1016/j.ecolmodel.2013.04.020 
Gastón, A., Garcia-Vinas, J. I., Bravo-Fernandez, A. J., López-Leiva, C., Oliet, J. A., Roig, S., \& Serrada, R. (2014). Species distribution models applied to plant species selection in forest restoration: are model predictions comparable to expert opinion? New forests, 45(5), 641-653. https://doi.org/10.1007/s11056014-9427-7

Gastón, A., \& García-Viñas, J. I. (2010). Updating coarse-scale species distribution models using small fine-scale samples. Ecological Modelling, 221(21), 2576-2581. https://doi.org/10.1016/j.ecolmodel.2010.07.016

Gelviz-Gelvez, S. M., Pavón, N. P., Illoldi-Rangel, P., \& Ballesteros-Barrera, C. (2015). Ecological niche modeling under climate change to select shrubs for ecological restoration in Central Mexico. Ecological Engineering, 74, 302-309. https://doi.org/10.1016/j.ecoleng.2014.09.082

Giacomin, L. L., Kamino, L. H. Y., \& Stehmann, J. R. (2014). Speeding up the discovery of unknown plants: a case study of Solanum (Solanaceae) endemics from the Brazilian Atlantic Forest. Boletim do Museu de Biologia Mello Leitão. Nova Série, 35, 121-135.

Guisan, A., \& Thuiller, W. (2005). Predicting species distribution: offering more than simple habitat models. Ecology Letters, 8(9), 993-1009. DOI: 10.1111/j.1461-0248.2005.00792.x

Guisan, A., Tingley, R., Baumgartner, J. B., Naujokaitis-Lewis, I., Sutcliffe, P. R., Tulloch, A. I., ... \& Buckley, Y. M. (2013). Predicting species distributions for conservation decisions. Ecology letters, 16(12), 1424-1435. https://doi.org/10.1111/ele.12189

Gutiérrez, J. M. G., \& Palomares, O. S. (1994). Estaciones ecológicas de los pinares españoles. Instituto Nacional para la Conservación de la Naturaleza

Harrell, F. E. (2001) Regression modeling strategies: with applications to linear models, logistic regression and survival analysis. Springer, New York.

Harris, J. A., Hobbs, R. J., Higgs, E., \& Aronson, J. (2006). Ecological restoration and global climate change. https://doi.org/10.1111/j.1526100X.2006.00136.X

Heringer, G., Bueno, M. L., Meira-Neto, J. A., Matos, F. A., \& Neri, A. V. (2019). Can Acacia mangium and Acacia auriculiformis hinder restoration efforts in the Brazilian Atlantic Forest under current and future climate conditions? Biological Invasions, 21(9), 2949-2962. https://doi.org/10.1007/s10530-01902024-7

Hulme P. E. (2009) Trade, transport and trouble: managing invasive species pathways in an era of globalization. Journal of Applied Ecology, 46:10-18. https://doi.org/10.1111/j.1365-2664.2008.01600.x

Kamino, L. H., Stehmann, J. R., Amaral, S., De Marco Jr, P., Rangel, T. F., de Siqueira, M. F., ... \& Hortal, J. (2012). Challenges and perspectives for species distribution modelling in the neotropics. Biology Letters, 8:324-326. https://doi.org/10.1098/rsbl.2011.0942

Lehmann, J. R., Prinz, T., Ziller, S. R., Thiele, J., Heringer, G., Meira-Neto, J. A., \& Buttschardt, T. K. (2017). Open-source processing and analysis of aerial imagery acquired with a low-cost unmanned aerial system to support invasive plant management. Frontiers in Environmental Science, 5, 44. https://doi.org/10.3389/fenvs.2017.00044

Lemes, P., Melo, A. S., \& Loyola, R. D. (2014). Climate change threatens protected areas of the Atlantic Forest. Biodiversity and Conservation, 23(2), 357368. https://doi.org/10.1007/s10531-013-0605-2

Martínez-Ramos, M., Pingarroni, A., Rodríguez-Velázquez, J., Toledo-Chelala, L., Zermeño-Hernández, I., \& Bongers, F. (2016). Natural forest regeneration and ecological restoration in human-modified tropical landscapes. Biotropica, 48(6), 745-757. https://doi.org/10.1111/btp.12382

Mateo, R. G., Gastón, A., María José, A.-F., Juan Ignacio, G.-V., \& Saura, S. (2018) Sampling strategies and optimization of species distribution modelling at the landscape scale. Forest Ecology and Management, 410: 104-113. https://doi.org/10.1016/j.foreco.2017.12.046

Meira-Neto, J. A. A., da Silva, M. C. N. A., Tolentino, G. S., Gastauer, M., Buttschardt, T., Ulm, F., \& Máguas, C. (2018). Early Acacia invasion in a sandy ecosystem enables shading mediated by soil, leaf nitrogen and facilitation. Biological Invasions, 20(6), 1567-1575. https://doi.org/10.1007/s10530-017-1647-2

Melo, F. P., Pinto, S. R., Brancalion, P. H., Castro, P. S., Rodrigues, R. R., Aronson, J., \& Tabarelli, M. (2013). Priority setting for scaling-up tropical forest restoration projects: Early lessons from the Atlantic Forest Restoration Pact. Environmental Science \& Policy, 33, 395-404. https://doi.org/10.1016/j.envsci.2013.07.013

Millar, C. I., Stephenson, N. L., \& Stephens, S. L. (2007) Climate change and forests of the future: managing in the face of uncertainty. Ecological Applications, 17, 2145-2151.

Oliveira, U., Paglia, A. P., Brescovit, A. D., de Carvalho, C. J., Silva, D. P., Rezende, D. T., ... \& Santos, A. J. (2016). The strong influence of collection bias on biodiversity knowledge shortfalls of Brazilian terrestrial biodiversity. Diversity and Distributions, 22(12), 1232-1244. https://doi.org/10.1111/ddi.12489

Oliveira, U., Soares-Filho, B. S., Paglia, A. P., Brescovit, A. D., De Carvalho, C. J., Silva, D. P., ... \& Santos, A. J. (2017). Biodiversity conservation gaps in the Brazilian protected areas. Scientific Reports, 7(1), 1-9. https://doi.org/10.1038/s41598-017-08707-2

Pemán García, J., Navarro Cerrillo, R. M., \& Serrada Hierro, R. (2006). Species selection guidelines in reforestation. Ruiz de la Torre's contributions. Investigación Agraria. Sistemas y Recursos Forestales (España). f 15:87-102.

Peterson, A. T., Soberón, J., Pearson, R. G., Anderson, R. P., Martínez-Meyer, E., Nakamura, M., \& Araújo, M. B. (2011). Ecological niches and geographic distributions (MPB-49). Princeton University Press.

Phillips, S. J., Anderson, R. P., \& Schapire, R. E. (2006). Maximum entropy modeling of species geographic distributions. Ecological Modelling, 190(3-4), 231-259. https://doi.org/10.1016/j.ecolmodel.2005.03.026

Pickering, C., \& Byrne, J. (2014). The benefits of publishing systematic quantitative literature reviews for PhD candidates and other early-career researchers. Higher Education Research \& Development, 33(3), 534-548. https://doi.org/10.1080/07294360.2013.841651 
Research, Society and Development, v. 10, n. 8, e46610817158, 2021

(CC BY 4.0) | ISSN 2525-3409 | DOI: http://dx.doi.org/10.33448/rsd-v10i8.17158

Ravenscroft, C., Scheller, R. M., Mladenoff, D. J., \& White, M. A. (2010). Forest restoration in a mixed-ownership landscape under climate change. Ecological Applications, 20(2), 327-346. https://doi.org/10.1890/08-1698.1

Renton, M., Shackelford, N., \& Standish, R. J. (2012). Habitat restoration will help some functional plant types persist under climate change in fragmented landscapes. Global Change Biology, 18(6), 2057-2070. https://doi.org/10.1111/j.1365-2486.2012.02677.x

Richardson, D. M., \& Rejmánek, M. (2011). Trees and shrubs as invasive alien species-a global review. Diversity and distributions, 17(5), 788-809. https://doi.org/10.1111/j.1472-4642.2011.00782.x

Ser. The ser international primer on ecological restoration. Society for Ecological Restoration International, Tucson - USA, 2004.

Team, R. C. (2020). R: A language and environment for statistical computing.

Torraco, R. J. (2016). Writing integrative literature reviews: Using the past and present to explore the future. Human resource development review, 15(4), 404428. https://doi.org/10.1177/1534484316671606

Tulloch, A. I. T., Sutcliffe, P., Naujokaitis-Lewis, I., Tingley, R., Brotons, L., Ferraz, K., Possingham, H., Guisan, A., \& Rhodes, J. R. (2016) Conservation planners tend to ignore improved accuracy of modelled species distributions to focus on multiple threats and ecological processes. Biological Conservation 199: 157-171. https://doi.org/10.1016/j.biocon.2016.04.023

Van Loon, A. H., Soomers, H., Schot, P. P., Bierkens, M. F. P., Griffioen, J., \& Wassen, M. J. (2011) Linking habitat suitability and seed dispersal models in order to analyse the effectiveness of hydrological fen restoration strategies. Biological Conservation 144: 1025-1035. https://doi.org/10.1016/j.biocon.2010.12.021 